\title{
Continuous Cardiac Output Measurement during Off-Pump Coronary Artery Bypass Grafting: Radial Arterial Pulse Wave versus Femoral Arterial Pulse Wave
}

\author{
Muralidhar Kanchi ${ }^{1}$ Manjunath Rudresh ${ }^{1} \quad$ Sucharita Das ${ }^{1}$ \\ ${ }^{1}$ Department of Anesthesia and Intensive Care, Narayana Institute of \\ Cardiac Sciences, Bangalore, Karnataka, India

\begin{abstract}
Address for correspondence Muralidhar Kanchi, MD, FIACTA, FICA, MBA Department of Anesthesia and Intensive Care, University of Minnesota, Narayana Hrudayalaya, \#258/A, Bommasandra Industrial Area, Anekal Taluk, Bangalore 560 099, Karnataka, India (e-mail: muralidhar.kanchi. dr@nhhospitals.org; kanchirulestheworld@gmail.com).
\end{abstract}

\begin{abstract}
Keywords

- cardiac output

- arterial pressure monitoring

- pulse contour analysis

Background and Objectives Measurement of cardiac output (CO) during off-pump coronary artery bypass grafting (OP-CABG) is useful to assess the impact of displacement of heart during surgery. FloTrac uses the pulse-contour analysis algorithm to derive stroke volume (SV) and CO. This study was aimed to determine whether the site of monitoring of arterial pressure influences the $\mathrm{CO}$ measurement using Flotrac/Vigileo. Methods The authors recorded 75 sets of observations in 15 patients who underwent elective OP-CABG. Each patient had the radial and femoral artery cannulated and connected to FloTrac/Vigileo. The hemodynamic parameters were monitored at specified points of time intraoperatively.

Results The CO, cardiac index, SV, stroke volume index, and stroke volume variation measured from radial artery did not differ from those of femoral artery at all points of measurement.

Conclusion Either of the sites of monitoring of arterial pressure (namely radial and femoral artery) is acceptable for monitoring CO during OP-CABG.
\end{abstract}

\section{Introduction}

Off-pump coronary artery bypass grafting (OP-CABG) has revolutionized the surgical approach for the treatment of coronary artery disease. OP-CABG avoids the use of extracorporeal circulation, and hence all the detrimental effects of instituting cardiopulmonary bypass (CPB), especially the systemic inflammatory response syndrome, are eliminated in patients who undergo this technique of coronary revascularization. Essential to the performance of OP-CABG is the use of mechanical stabilizers, for example octopus II and/ or starfish, which make the distal anastomotic site immobile during grafting. In addition to mechanical stabilization, man ipulation of the heart to provide access to the distal anastomotic site is necessary for optional visualization. Use of mechanical stabilizers and manipulation of the heart for optimal position prior to grafting makes the hemodynamic state unsteady and is associated with fall in cardiac output (CO) and systemic hypotension. Hemodynamic assessment involving $\mathrm{CO}$ measurement is of particular importance in patients undergoing OP-CABG. The intermittent thermodilution bolus techniques using a pulmonary artery catheter is still accepted as a standard method for measurement of $\mathrm{CO}$, despite its invasive character and associated risks. Recently, less invasive methods and hardware to assess CO have become available. One of these alternatives is the FloTrac/Vigileo system (Edwards Lifesciences), which determines CO by analyzing the peripheral arterial waveform of an artery. FloTrac is easy to install, safe to use, and does not need any specific catheter or external calibration. FloTrac is based on the following equation: stroke volume $(\mathrm{SV})=$ pulsatility $\times$ kappa. Pulsatility is calculated by using the standard deviation (SD) of a peripheral arterial pressure wave. Kappa is a number that represents the vascular tone of the patient's arterial tree, and it takes into account the age, weight, height, and sex.
Dol https://doi.org/ $10.1055 / \mathrm{s}-0038-1624062$ ISSN 2457-0206.
Copyright @ 2017 Official Publication of The Simulation Society (TSS), accredited by International Society of Cardiovascular Ultrasound (ISCU)
License terms

(1) (1) $\Theta \circledast$ 
The FloTrac sensor has been proven accurate and reliable in monitoring $\mathrm{CO}$ in clinical studies when compared against continuous cardiac output (CCO) and intermittent cardiac output (ICO). ${ }^{1}$ A prior study found satisfactory agreement of the new device with intermittent and continuous thermodilution. $^{2}$ Good agreement was found between CO values obtained by arterial pulse contour analysis and thermodilution technique using PAC in the intraoperative period in patients undergoing OP-CABG. ${ }^{3}$ Rose-Marieke have demonstrated that $\mathrm{CO}$ measured by a new arterial pressure waveform analysis using FloTrac/Vigileo is clinically applicable after cardiac surgery and performs well at low $\mathrm{CO}^{4}$

Physiologically speaking, arterial pressure measured in a more peripheral artery is different from the central aortic pressure, because the arterial waveform becomes progressively more distorted as the signal is transmitted down the arterial system. The high-frequency components, such as the dicrotic notch, disappear, the systolic peak increases, the diastolic trough decreases, and there is a transmission delay. ${ }^{5}$ These changes are caused by decrement in arterial compliance as we progress to periphery and reflection/resonance of pressure waves in the arterial tree. However, despite this distortion, the mean arterial pressure (MAP) measured in the peripheral artery is similar to the central aortic pressure under normal circumstances. So far, few studies have examined the impact of the site of arterial cannulation on the accuracy of the CO measurement using the FloTrac/ Vigileo system in patients undergoing OP-CABG. This study was performed to evaluate whether $\mathrm{CO}$ measurement at two different sites of arterial cannulation namely radial and femoral will influence the determination and whether the two sites can be used interchangeably in terms of monitoring $\mathrm{CO}$ in patients undergoing OP-CABG.

\section{Methods}

After obtaining institutional review board (IRB) approval, ethical clearance, and written informed consent, 15 adult patients scheduled for elective CABG without the use of extracorporeal circulation (OP-CABG) were studied. Those patients with permanent pacemakers, need for mechanical cardiac support, severe peripheral vascular disease, and valvular dysfunction were excluded from the study. All patients underwent thorough preoperative examination and evaluation, which included echocardiography and coronary angiography. Preanesthetic medication consisted of 5 to $10 \mathrm{mg}$ of oral diazepam the night prior and on the morning of surgery. All antihypertensive and antianginal medication was continued till the day of surgery with the exception of angiotensin-converting enzyme inhibitors and angiotensin-receptor blockers. After establishment of peripheral venous access, the right radial artery and right femoral artery were cannulated using 20G arterial cannula and 18G cannula, respectively, under local analgesia after supplemental sedation while patients breathed oxygen $\left(\mathrm{O}_{2}\right)$ though facemask. If the right radial/femoral artery had been utilized for the preoperative coronary angiography, contralateral radial/femoral artery was cannulated. Both the arterial lines were simultaneously connected via a dedicated FloTrac pressure transducer (-Figs. 1-3) to Vigileo monitor (Edwards Lifesciences; software version 1.14, with improved automatic vascular tone adjustment 1 minute averaging with added fluid optimization screen enhancements and broader patient range to include high-risk surgical patients).

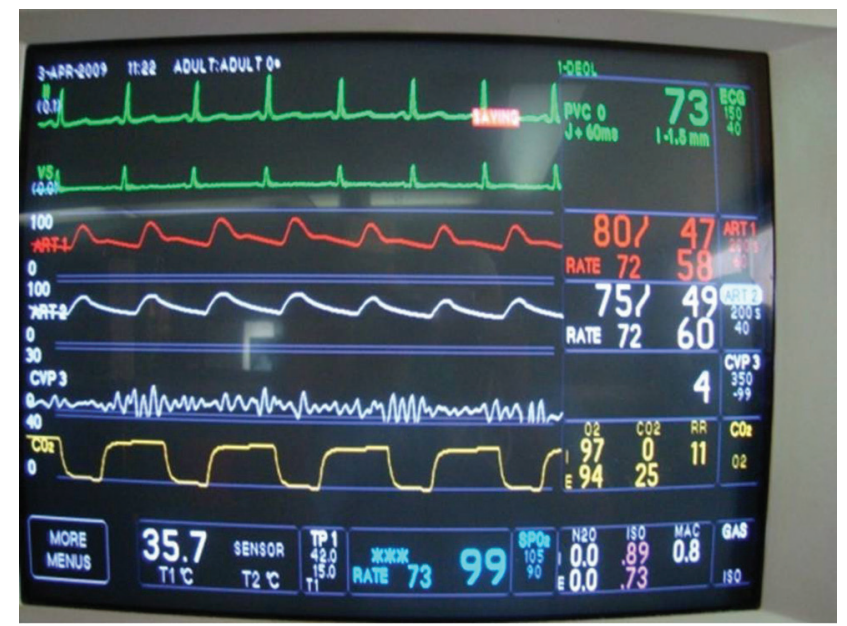

Fig. 1 Simultaneous monitoring of radial and femoral arterial pressure through FloTrac.

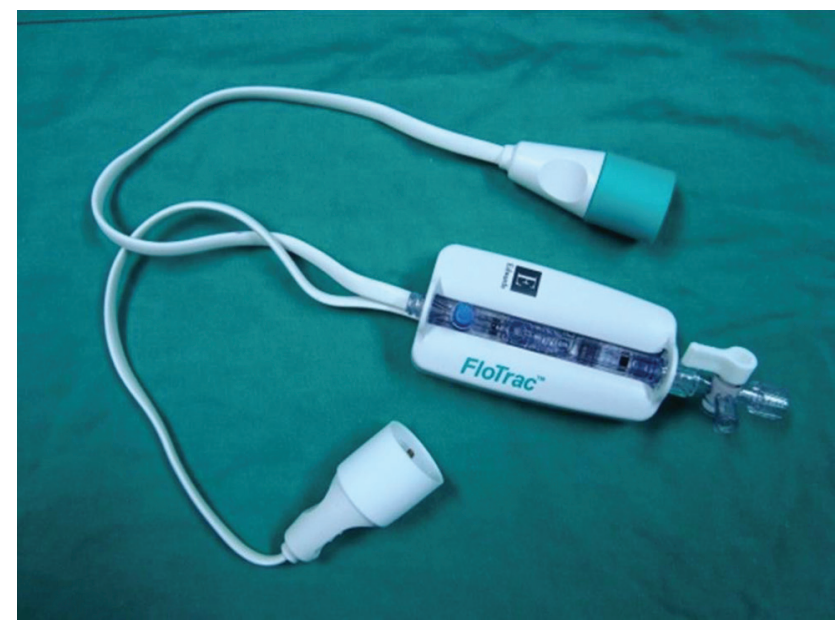

Fig. 2 Picture of FloTrac transducer

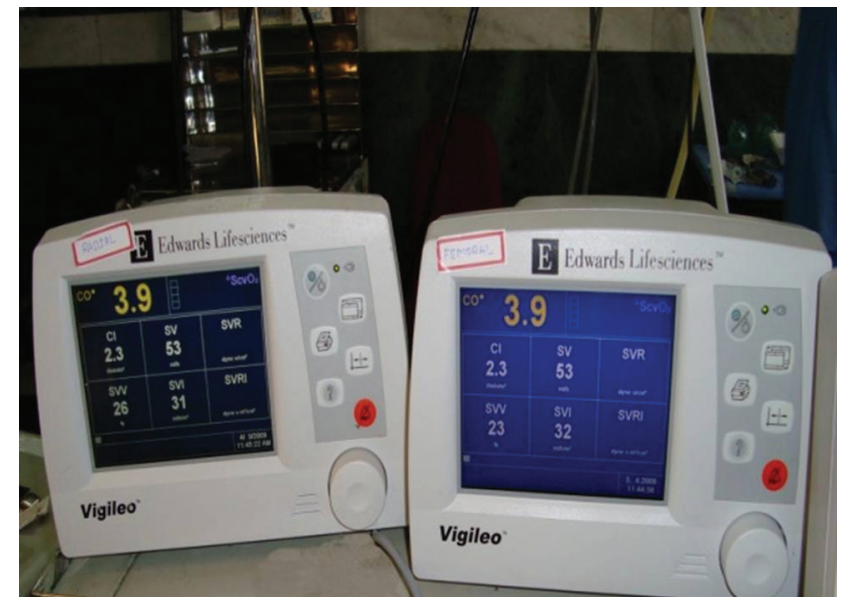

Fig. 3 Simultaneous monitoring of cardiac output/cardiac index, SVV, and SV for radial and femoral arteries using FloTrac/Vigileo. 
After intubation of the trachea, the lungs were ventilated with $50 \%$ oxygen in air and isoflurane. Ventilation was controlled with a tidal volume of $10 \mathrm{~mL} / \mathrm{kg}$, and the ventilatory rate was adjusted to maintain an arterial partial pressure of carbon dioxide $\left(\mathrm{CO}_{2}\right)$ of 32 to $42 \mathrm{~mm} \mathrm{Hg}$ and arterial $\mathrm{pH}$ between 7.35 and 7.45 . The heart rate and arterial blood pressure were maintained within $20 \%$ of basal values. The central venous pressure was maintained between 10 and 14 $\mathrm{mm} \mathrm{Hg}$. OP-CABG was done after median sternotomy under normothermic conditions with $\mathrm{CPB}$ standby. Heparin was used in a dose of $400 \mathrm{U} / \mathrm{kg}$ to maintain activated coagulation time (ACT) of at least 400 seconds. Proximal anastomosis was done with aortic partial clamping, and distal grafting was performed using octopus-II tissue stabilizer. OP-CABG was converted to conventional CABG with $\mathrm{CPB}$, if the patient could not tolerate the procedure off-pump. After anastomoses, heparin was neutralized with protamine in a ratio of $1 \mathrm{mg}$ protamine for every 100 units of initial dose of heparin. No antifibrinolytic agents were used. Blood and blood products were not used until hematocrit was less than $27 \%$. Patients were electively ventilated at the end of surgery, and early extubation protocol was applied to all patients.

Postoperatively, all patients were transferred to the intensive care unit (ICU), and controlled mechanical ventilation was continued for at least 4 hours. Peak airway pressures were adjusted to deliver a constant tidal volume of $10 \mathrm{~mL} / \mathrm{kg}$ body weight; inspiratory-expiratory time ratio was set to $1: 2$, and a positive end-expiratory pressure (PEEP) of $5 \mathrm{~mm} \mathrm{Hg}$ was used. Sedation consisted of a continuous propofol infusion of 0.5 to $1.5 \mathrm{mg} / \mathrm{kg} / \mathrm{h}$. Tracheal extubation was performed when hemodynamics were stable, body temperature was greater than $36^{\circ} \mathrm{C}$, patients breathed spontaneously with adequate blood gas variables, and there was no excessive bleeding.
The measurements and the data collection were performed by an independent observer who was not involved in the anesthesia care of the patients at 11 different intraoperative time points. The measurements were recorded during a hemodynamically stable phase. The measurements were recorded at the following: (1) prior to anesthesia induction, (2) after anesthetic induction, (3) after endotracheal intubation, (4) at skin inclusion, (5) sternotomy, (6) during left internal mammary artery (LIMA) harvest, (7) during first distal grafting, (8) during second distal grafting, (9) during proximal anastomosis, (10) after grafting, and (11) at skin closure. Data were expressed as mean (SD) and subjected to statistical analysis using one-way analysis of variance (ANOVA) for comparing radial with femoral data over time.

\section{Results}

Fifteen patients were studied, of whom 13 were male. The mean age was $52.8 \pm 11.8(\mathrm{SD})$ years and weight $67.7 \pm 10.6$ (SD) kg. Complete basic demographic details are presented in - Table 1. The mean left ventricular (LV) ejection fraction was $53 \% \pm 5.6 \%$ (SD). - Table2 shows the means values of the hemodynamic variables in two arterial systems. Though the radial artery systolic pressure was higher than that of femoral artery, there were no differences in any other parameter at any point of measurement. The $\mathrm{CO}$, cardiac index $(\mathrm{CI})$, stroke volume variation (SVV), SV, and stroke volume index (SVI) were similar at both sites of measurement.

\section{Discussion}

Arterial pressure waveform analysis to determine $\mathrm{CO}$ has already been introduced into clinical practice. Commercially

Table 1 Basic demographics of the study patients

\begin{tabular}{|l|l|l|l|l|l|l|l|l|l|l|}
\hline $\begin{array}{l}\text { SI. } \\
\text { No. }\end{array}$ & $\begin{array}{l}\text { Age } \\
(\mathrm{y})\end{array}$ & Sex & DM & HTN & EF & $\begin{array}{l}\text { SCr } \\
(\mathbf{m g})\end{array}$ & $\begin{array}{l}\text { IPPV } \\
(\mathbf{m i n})\end{array}$ & $\begin{array}{l}\text { ICU stay } \\
(\mathbf{d})\end{array}$ & $\begin{array}{l}\text { Hospital } \\
\text { stay (d) }\end{array}$ & Others \\
\hline 1 & 37 & $\mathrm{M}$ & $\mathrm{Y}$ & $\mathrm{Y}$ & 60 & 0.9 & 11.3 & 2 & 12 & Nil \\
\hline 2 & 49 & $\mathrm{M}$ & $\mathrm{N}$ & $\mathrm{N}$ & 45 & 0.8 & 16.3 & 2 & 9 & Smoker, dyslipidemia \\
\hline 3 & 39 & $\mathrm{M}$ & $\mathrm{N}$ & $\mathrm{Y}$ & 55 & 1 & 25.45 & 7 & 19 & Nil \\
\hline 4 & 48 & $\mathrm{M}$ & $\mathrm{N}$ & $\mathrm{Y}$ & 60 & 1 & 19.45 & 2 & 8 & Asthma \\
\hline 5 & 45 & $\mathrm{M}$ & $\mathrm{N}$ & $\mathrm{N}$ & 55 & 0.8 & 24.15 & 2 & 7 & Smoker \\
\hline 6 & 70 & $\mathrm{~F}$ & $\mathrm{~N}$ & $\mathrm{~N}$ & 60 & 0.7 & 18.45 & 2 & 10 & Nil \\
\hline 7 & 47 & $\mathrm{M}$ & $\mathrm{Y}$ & $\mathrm{Y}$ & 45 & 0.6 & 24 & 2 & 15 & $\begin{array}{l}\text { Asthmatic } \\
\text { H/o syncope }\end{array}$ \\
\hline 8 & 57 & $\mathrm{M}$ & $\mathrm{Y}$ & $\mathrm{Y}$ & 60 & 1 & 23 & 6 & 16 & Nil \\
\hline 9 & 68 & $\mathrm{M}$ & $\mathrm{Y}$ & $\mathrm{N}$ & 52 & 1 & 72 & 4 & 10 & Nil \\
\hline 10 & 72 & $\mathrm{M}$ & $\mathrm{N}$ & $\mathrm{Y}$ & 55 & 1.3 & 17 & 2 & 8 & Nil \\
\hline 11 & 55 & $\mathrm{M}$ & $\mathrm{Y}$ & $\mathrm{Y}$ & 40 & 0.9 & 16.5 & 2 & 7 & Nil \\
\hline 12 & 58 & $\mathrm{M}$ & $\mathrm{Y}$ & $\mathrm{Y}$ & 53 & 0.9 & 23 & 4 & 9 & Smoker \\
\hline 13 & 63 & $\mathrm{M}$ & $\mathrm{N}$ & $\mathrm{N}$ & 55 & 1.6 & 22.3 & 3 & 14 & Smoker \\
\hline 14 & 59 & $\mathrm{~F}$ & $\mathrm{Y}$ & $\mathrm{N}$ & 60 & 0.8 & 20 & 3 & 11 & Nil \\
\hline 15 & 65 & $\mathrm{M}$ & $\mathrm{Y}$ & $\mathrm{Y}$ & 50 & 1 & 18.3 & 3 & 12 & Smoker \\
\hline
\end{tabular}

Abbreviations: DM, diabetes mellitus; EF, ejection fraction; F, female; HTN, hypertension; ICU, intensive care unit; IPPV, intermittent positive-pressure ventilation; $\mathrm{M}$, male; $\mathrm{N}$, no; $\mathrm{SCr}$, serum creatinine; $\mathrm{Y}$, yes. 


\begin{tabular}{|c|c|c|c|c|c|c|c|c|c|c|c|c|c|}
\hline \multirow{2}{*}{$\begin{array}{l}\frac{\tilde{N}}{E} \\
\frac{\vec{E}}{E} \\
\bar{\Sigma} \\
\vec{n}\end{array}$} & $\begin{array}{l}\bar{T} \\
\frac{0}{0} \\
\frac{E}{4} \\
4\end{array}$ & $\begin{array}{l}\text { in } \\
+1 \\
\text { in }\end{array}$ & $\begin{array}{l}+1 \\
\infty \\
\stackrel{+}{+} \leftarrow \\
\dot{\sigma}\end{array}$ & $\begin{array}{l}\infty \\
+1 \\
\infty \\
\dot{\sigma}\end{array}$ & $\begin{array}{l}+1 \\
\stackrel{+}{*} \\
\stackrel{\sim}{\sigma}\ulcorner\end{array}$ & 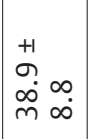 & \begin{tabular}{l}
$n$ \\
+1 \\
$\check{+}$ \\
\multirow{\gamma}{*}{}
\end{tabular} & 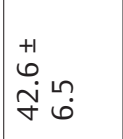 & 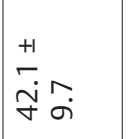 & 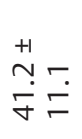 & 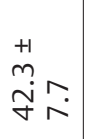 & $\begin{array}{l}+1 \\
\stackrel{+}{\dot{b}} \widetilde{i} \\
\dot{\varphi}\end{array}$ & $\frac{\ddot{\oplus}}{\stackrel{\Xi}{\Xi}}$ \\
\hline & $\begin{array}{l}\overline{\underline{\pi}} \\
\frac{\pi}{\pi} \\
\propto\end{array}$ & 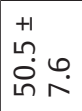 & $\begin{array}{ll}+1 & \infty \\
\stackrel{+}{+} & \infty\end{array}$ & $\begin{array}{l}+1 \\
m \\
\dot{q} \\
\dot{q}\end{array}$ & $\begin{array}{l}\stackrel{+1}{\sim} \\
\stackrel{\infty}{\sim} \\
\stackrel{\infty}{r}\end{array}$ & $\begin{array}{ll}\infty & a \\
\dot{m} & \sigma \\
m & +1\end{array}$ & $\begin{array}{ll}m & 0 \\
\dot{q} & 0 \\
+1\end{array}$ & $\begin{array}{l}+1 \\
\underline{\varphi} \\
\stackrel{m}{m} \dddot{r}\end{array}$ & $\begin{array}{ll}+1 & N \\
o & 0 \\
f & 0\end{array}$ & \begin{tabular}{l}
$a$ \\
+1 \\
\multirow{7}{*}{}
\end{tabular} & $\begin{array}{l}+1 \\
m \\
\dot{m}_{m} \\
m \\
\infty\end{array}$ & 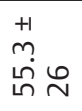 & $n^{n}$ \\
\hline \multirow{2}{*}{$\begin{array}{l}\frac{\partial}{\vec{E}} \\
\frac{\vec{n}}{n}\end{array}$} & $\begin{array}{l}\overline{0} \\
\frac{0}{0} \\
\frac{1}{4} \\
\stackrel{4}{1}\end{array}$ & $\begin{array}{l}6 \\
+1 \\
6 \\
\infty\end{array}$ & $\begin{array}{l}+1 \\
\stackrel{+}{r} \hat{r} \\
\dot{r}\end{array}$ & 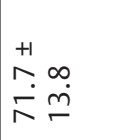 & $\begin{array}{l}+1 \\
\stackrel{+}{n} \\
\stackrel{n}{r} \\
\infty\end{array}$ & $\begin{array}{l}+1 \\
\stackrel{0}{0} \\
\dot{\theta} \sim\end{array}$ & 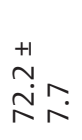 & $\begin{array}{l}+1 \\
\stackrel{+}{*} \kappa \\
\stackrel{\pi}{\pi}\end{array}$ & $\begin{array}{ll}+1 & \infty \\
\Gamma & \stackrel{+}{\leftarrow}\end{array}$ & $\begin{array}{l}+1 \\
\stackrel{+}{N} \sim \\
\stackrel{\nabla}{\sim} \infty \\
\Gamma\end{array}$ & 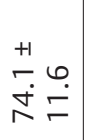 & 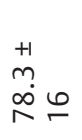 & 8 \\
\hline & $\begin{array}{l}\overline{\frac{\pi}{7}} \\
\frac{\pi}{\alpha} \\
\alpha\end{array}$ & $\begin{array}{l}\stackrel{+1}{N} \\
\stackrel{\dot{\infty}}{\infty} \pm\end{array}$ & 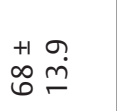 & 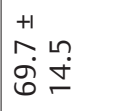 & 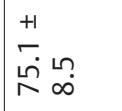 & 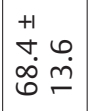 & 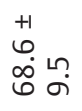 & 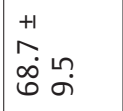 & $\stackrel{+1}{m} \underset{\hat{\sigma}}{\stackrel{\Gamma}{\tau}}$ & 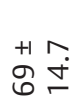 & 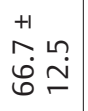 & 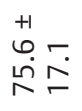 & 芩 \\
\hline \multirow{2}{*}{$\stackrel{\overbrace{}}{Ð}$} & $\begin{array}{l}\bar{T} \\
\text { 은 } \\
\frac{1}{4}\end{array}$ & 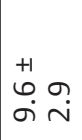 & $\begin{array}{l}+1 \\
\infty \\
0 \\
0 \\
+ \\
\llcorner\end{array}$ & $\begin{array}{l}\text { nn } \\
+1 \\
m \\
\check{-}\end{array}$ & 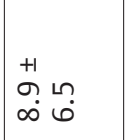 & 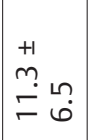 & $\begin{array}{l}n \\
\varphi \\
+1 \\
\infty\end{array}$ & $\begin{array}{l}+1 \\
\stackrel{+}{\sim} \sim \\
\mp \infty\end{array}$ & $\begin{array}{l}+1 \\
6 \\
\infty \\
\infty\end{array}$ & 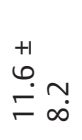 & $\begin{array}{l}+1 \\
\dot{\sigma} \\
\sigma \\
\dot{\sigma}\end{array}$ & $\begin{array}{ll}+1 & 0 \\
m & 0 \\
\sigma & 0\end{array}$ & 言 \\
\hline & $\begin{array}{l}\bar{\pi} \\
\frac{\pi}{\overline{2}} \\
\frac{\pi}{\alpha}\end{array}$ & $\begin{array}{ll}+1 & \\
\infty & 0 \\
0 & 0 \\
- & \dot{m}\end{array}$ & 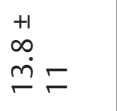 & 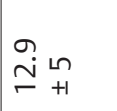 & 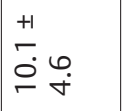 & $\begin{array}{ll}+1 & - \\
\sigma & \text { டे }\end{array}$ & $\stackrel{+H}{\stackrel{H}{r}} \stackrel{+}{m}$ & $\stackrel{+1}{\stackrel{+}{\sigma}} \dot{m}$ & 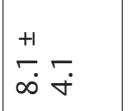 & $\begin{array}{ll}+1 & \\
\infty & \\
\stackrel{+}{+} & \stackrel{2}{\infty} \\
\infty\end{array}$ & 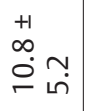 & 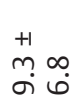 & $\bar{C}$ \\
\hline \multirow{2}{*}{ 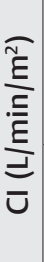 } & $\begin{array}{l}\bar{\pi} \\
\frac{\pi}{0} \\
\frac{1}{0} \\
\sqcup\end{array}$ & $\begin{array}{l}n \\
0 \\
+1 \\
+1 \\
\sim \\
m\end{array}$ & $\begin{array}{l}\hat{0} \\
+1 \\
\sigma \\
\sim\end{array}$ & $\begin{array}{l}\tilde{0} \\
0 \\
+1 \\
+\dot{m} \\
\dot{m}\end{array}$ & 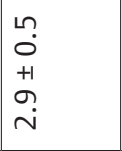 & 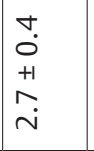 & $\begin{array}{l}+ \\
0 \\
+1 \\
m\end{array}$ & $\begin{array}{l}\dot{+} \\
\dot{+} \\
+1 \\
\dot{n}\end{array}$ & $\begin{array}{l}\tilde{0} \\
0 \\
+1 \\
\sim \\
m\end{array}$ & $\begin{array}{l}\hat{0} \\
+1 \\
\infty \\
\sim \\
\sim\end{array}$ & $\begin{array}{l}9 \\
0 \\
+1 \\
r \\
m\end{array}$ & $\begin{array}{l}\tau \\
\dot{0} \\
+1 \\
\dot{m} \\
\dot{m}\end{array}$ & 5 \\
\hline & $\begin{array}{l}\bar{\pi} \\
\frac{\pi}{\pi} \\
\check{\alpha}\end{array}$ & $\stackrel{+1}{-} \stackrel{t}{m}$ & $\stackrel{+1}{i} \hat{0}$ & $\begin{array}{ll}+1 & \\
& 0 \\
m & 0\end{array}$ & $\begin{array}{l}+1 \\
\infty \\
\sim \\
\sim\end{array}$ & $\begin{array}{lll}+1 & \\
& \text { ? } \\
& 0\end{array}$ & $\begin{array}{ll}+1 & \\
\text { ô } & \text { ! } \\
\sim & 0\end{array}$ & 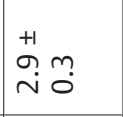 & 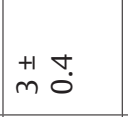 & 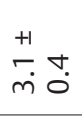 & 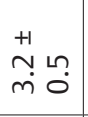 & $\begin{array}{ll}+1 & N \\
m & 0\end{array}$ & 8 \\
\hline \multirow{2}{*}{ 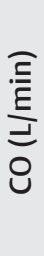 } & 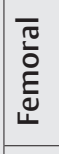 & $\begin{array}{l}9 \\
0 \\
+1 \\
+ \\
+ \\
\text { เn }\end{array}$ & $\begin{array}{l}m \\
\stackrel{n}{+} \\
+1 \\
\text { in }\end{array}$ & $\begin{array}{l}- \\
+1 \\
6 \\
\dot{1} \\
\dot{1}\end{array}$ & $\begin{array}{l}\infty \\
0 \\
+1 \\
+1 \\
\dot{1} \\
\text { in }\end{array}$ & $\begin{array}{l}0 \\
0 \\
+1 \\
\stackrel{+}{+} \\
\dot{\sigma}\end{array}$ & $\begin{array}{l}\infty \\
0 \\
+1 \\
+1 \\
\sim \\
\text { in }\end{array}$ & $\begin{array}{l}\sigma \\
0 \\
+1 \\
\dot{0} \\
\dot{0}\end{array}$ & 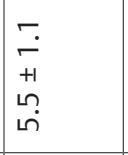 & $\begin{array}{l}m \\
\stackrel{m}{+} \\
+1 \\
\dot{v} \\
\dot{v}\end{array}$ & 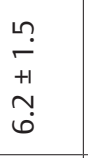 & 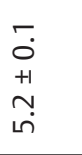 & 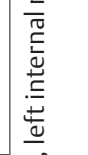 \\
\hline & $\begin{array}{l}\bar{\pi} \\
\frac{\pi}{\bar{\pi}} \\
\stackrel{2}{\alpha}\end{array}$ & $\begin{array}{ll}+1 \\
\stackrel{1}{*} \\
\stackrel{1}{n} & 0\end{array}$ & $\stackrel{+1}{n} \stackrel{m}{-}$ & 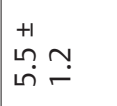 & $\begin{array}{l}+1 \\
\sigma \\
\sigma \\
\sigma\end{array}$ & $\begin{array}{ll}+1 & \\
\varphi & \infty \\
\dot{\sigma} & 0\end{array} \mid$ & $\begin{array}{l}- \\
+1 \\
\text { in }\end{array}$ & $\stackrel{+1}{+} \underset{\dot{1}}{\infty} \stackrel{\infty}{0}$ & 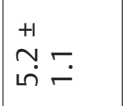 & $\begin{array}{l}\varphi \cdot- \\
\dot{\omega}++\end{array}$ & $\begin{array}{l}+1 \\
\omega \\
\dot{\omega} \\
-\end{array}$ & in $\stackrel{n}{-}$ & ثَثِ \\
\hline $\begin{array}{c}\text { Ỗ } \\
\text { T } \\
\underline{E} \\
\underline{E}\end{array}$ & $\begin{array}{l}\bar{\pi} \\
\overline{0} \\
\frac{0}{0} \\
4\end{array}$ & 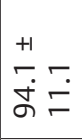 & $\begin{array}{l}+1 \infty \\
\stackrel{\infty}{N}\end{array}$ & 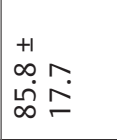 & $\begin{array}{ll}+1 & \ddots \\
\infty & \sigma\end{array}$ & $\begin{array}{l}+1 \\
\stackrel{+1}{\infty} \\
\infty \\
\infty\end{array}$ & 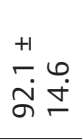 & $\begin{array}{ll}+1 & \\
\infty & \varrho \\
\infty & 0 \\
\infty & -\end{array}$ & $\begin{array}{l}\stackrel{\ominus}{1} \\
+1 \\
\sigma\end{array}$ & $\begin{array}{l}+1 \\
\stackrel{b}{\sigma}-\dot{\sigma} \\
\dot{\sigma} \sigma\end{array}$ & $\stackrel{+1}{\dot{+}} \underset{\sigma}{\sigma}$ & 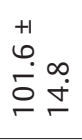 & $\begin{array}{l}\stackrel{\tilde{I}}{\subseteq} \\
\stackrel{a}{I}\end{array}$ \\
\hline 高 & $\begin{array}{l}\bar{\pi} \\
\frac{\pi}{\pi} \\
\check{\alpha}\end{array}$ & 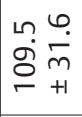 & $\begin{array}{l}+1 \\
m \\
m \\
\infty\end{array}$ & $\stackrel{+1}{\stackrel{+}{\sim}} \stackrel{+}{\infty}$ & 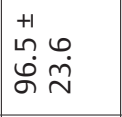 & 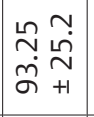 & $\stackrel{+1}{\circ}$ & $\begin{array}{ll}+1 & \\
\infty & - \\
\dot{0} & 0 \\
\infty & -\end{array}$ & m & 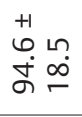 & $\begin{array}{ll}+1 \\
\dot{n} & \tilde{\sigma}\end{array}$ & $\begin{array}{ll}+1 & 0 \\
2 & \infty \\
\sigma & \stackrel{0}{-}\end{array}$ & 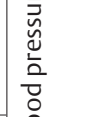 \\
\hline \multirow{2}{*}{ 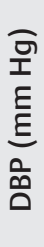 } & $\begin{array}{l}\bar{\pi} \\
\frac{0}{0} \\
\frac{E}{4} \\
1\end{array}$ & 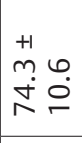 & 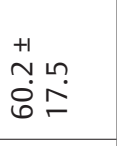 & $\stackrel{+1}{\check{\sigma}} \stackrel{\sim}{\sigma}$ & $\begin{array}{l}+1 \\
\text { in } \\
\check{n} \\
\end{array}$ & 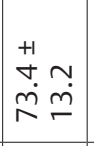 & 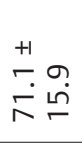 & 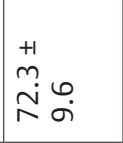 & 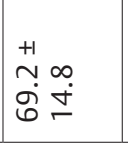 & 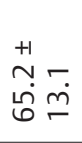 & $\begin{array}{l}+1 \\
\sim \\
\stackrel{n}{n} \sigma \\
\sigma\end{array}$ & 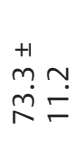 & 䒕 \\
\hline & 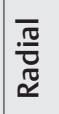 & $\stackrel{+1}{\hat{\infty}} \overline{\dot{N}}$ & 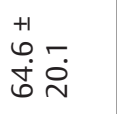 & 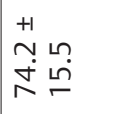 & $\stackrel{+1}{\stackrel{n}{\sim}} \stackrel{m}{\stackrel{\sim}{N}}$ & 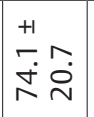 & $\begin{array}{ll}+1 & \infty \\
\infty & \stackrel{n}{m} \\
\infty & m\end{array}$ & 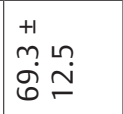 & $\stackrel{r}{r} \underset{r}{+}$ & 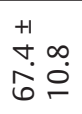 & $\stackrel{+}{r} \tilde{m}$ & $\begin{array}{l}+1 \\
\underline{\omega} \\
\dot{m} \\
\end{array}$ & 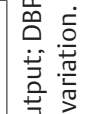 \\
\hline \multirow{2}{*}{ 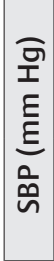 } & $\begin{array}{l}\bar{\pi} \\
\frac{0}{0} \\
\frac{1}{4}\end{array}$ & 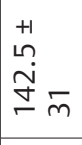 & 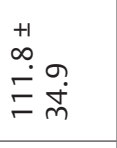 & 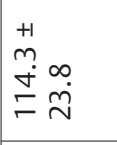 & 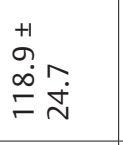 & 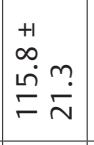 & 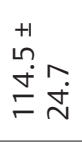 & 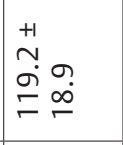 & 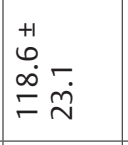 & $\begin{array}{l}\stackrel{+1}{\sim} \\
\stackrel{+}{\sim} \\
\stackrel{D}{\sim}\end{array}$ & 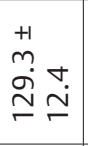 & $\begin{array}{ll}+1 & 0 \\
\stackrel{0}{m} & \dot{+} \\
-\end{array}$ & 恶 \\
\hline & 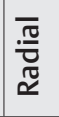 & 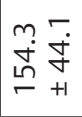 & 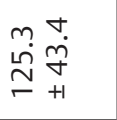 & 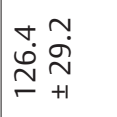 & $\begin{array}{ll}r & 0 \\
\dot{+} & \dot{v} \\
\sim & +1\end{array}$ & $\begin{array}{ll}N & 0 \\
& 0 \\
N & 0 \\
\sim & +1\end{array}$ & 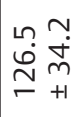 & 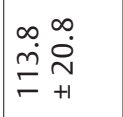 & 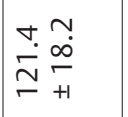 & $\begin{array}{l}\stackrel{+1}{b} m \\
\stackrel{n}{\sim} \underset{\sim}{N}\end{array}$ & 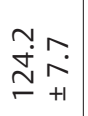 & $\stackrel{+1}{\stackrel{n}{n}} \bar{\sim}$ & $\hbar$ \\
\hline \multirow{2}{*}{\multicolumn{2}{|c|}{ 呈 }} & 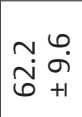 & $\stackrel{\infty}{\stackrel{\infty}{n}}$ & 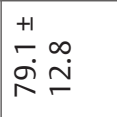 & $\begin{array}{l}n \\
\sigma \\
\sigma\end{array}$ & $\begin{array}{l}m \\
\stackrel{n}{r} \\
+1\end{array}$ & $\stackrel{m}{\stackrel{\sim}{\sim} \mp}$ & 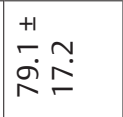 & $\stackrel{+1}{n} \stackrel{m}{N}$ & 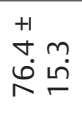 & $\left|\begin{array}{ll}+1 & - \\
\omega & 0 \\
\infty & -\end{array}\right|$ & 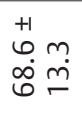 & 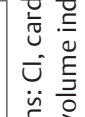 \\
\hline & & 充: & 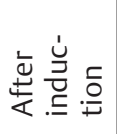 & 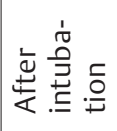 & 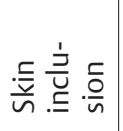 & 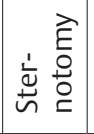 & $\sum_{\substack{\grave{n}\\
}}^{\grave{n}}$ & 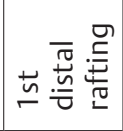 & 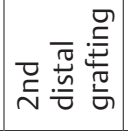 & $\begin{array}{l}\frac{1}{\grave{x}} \\
\stackrel{0}{a} \\
\stackrel{0}{E}\end{array}$ & 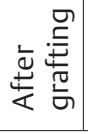 & 号 & 离 \\
\hline
\end{tabular}


wavailable devices include the PiCCO system (PiCCO, Pulsion Medical Systems) or the LiDCO system (LiDCO-plus, LiDCO Ltd.), which both require invasive calibration and recalibration by either transpulmonary thermodilution or lithium dilution after a certain time to compensate for interindividual differences in vascular compliance. ${ }^{6,7}$ The Modelflow technique (Finapres Medical Systems) requires calibration by thermodilution or determination of the aortic diameter. ${ }^{8}$ With pulse contour analysis, there are many issues such as vessel impedance, peripheral vascular resistance, and arterial compliance that are the basis of arterial waveform analysis for measuring $\mathrm{CO}^{9}$ Sources of error include interindividual differences in aortic impedance, an important variable to calculate SV, or reflections of the arterial pulse contour due to vessel tapering, bifurcations, and caliber changes, which may corrupt the peripheral arterial pulse wave signal. ${ }^{10,11} \mathrm{~A}$ recently developed device (FloTrac/Vigileo) offers the possibility of uncalibrated continuous CO measurements on the basis of arterial waveform analysis combined with simple usability. ${ }^{12,13}$ A recent software improvement of FloTrac consists of a more frequent recalculation of an internal variable estimating vascular tone combined with reduction in pulse wave detection noise.

The meta-analysis conducted by Mayer et al confirmed that FloTrac second generation is more accurate (lower bias) and more precise (lower percentage error) than FloTrac first generation. ${ }^{14}$ In a multicentric study of FloTrac versus esophageal Doppler to measure SV in patients undergoing surgery and critically ill patients in ICU, the authors showed that the FloTrac second generation is more precise and reduces percentage error. ${ }^{15} \mathrm{~A}$ yet another study assessing the $\mathrm{CO}$ charges showed that second-generation FlorTrac is more precise. ${ }^{16}$

The authors strongly feel that the CO measurement using pulmonary artery catheter (PAC) or transesophageal echocardiography (TEE) during an off-pump procedure when the heart is displaced is inaccurate for the following reasons: with PAC, the accuracy of the CO measurement is questionable when the heart is displaced as the catheter is likely to contact the right ventricular (RV) wall; hence, both intermittent and continuous CO measurements using thermodilution will be invalid during the period of cardiac displacement. In addition, the kinking of RV outflow tract will impede the free passage of the cold injectate. With TEE, the placement of swab behind the heart during LAD (left anterior descending artery) grafting and vertical displacement of the heart during OM (obtuse marginal artery) grafting will distort the image and accurate determination of the volumes for $\mathrm{CO}$ will not possible. Hence, during distal grafting in OP-CABG, there is a definite place for $\mathrm{CO}$ measurement based on pulse contour analysis as this is not rendered inaccurate by displacement/ virtualization of the heart or placement of swab behind the heart.

A recent study by Maddali et al has shown that both the radial $\mathrm{CO}$ and femoral $\mathrm{CO}$ measurements are reliable and comparable with the TEE measurements at various stages of cardiac surgery. ${ }^{17}$

\section{Conclusion}

This study demonstrates that CO measurement at either of the brachial artery or femoral artery sites pressure using FloTrac provides similar data in patients undergoing OP-CABG.

\section{Conflict of Interest}

All the authors have no conflict of interest.

\section{Funding Statement}

No funding was received for this study.

\section{Acknowledgments}

None.

\section{References}

1 Dr Gerry Manecke. Poster, SCCM 2005. Dr William McGee, Poster, ISICEM; 2005

2 Manecke GR Jr, Auger WR. Cardiac output determination from the arterial pressure wave: clinical testing of a novel algorithm that does not require calibration. J Cardiothorac Vasc Anesth 2007;21(1):3-7

3 Mehta Y, Chand RK, Sawhney R, Bhise M, Singh A, Trehan N. Cardiac output monitoring: comparison of a new arterial pressure waveform analysis to the bolus thermodilution technique in patients undergoing off-pump coronary artery bypass surgery. J Cardiothorac Vasc Anesth 2008;22(3):394-399

4 Breukers RM, Sepehrkhouy S, Spiegelenberg SR, Groeneveld AB. Cardiac output measured by a new arterial pressure waveform analysis method without calibration compared with thermodilution after cardiac surgery. J Cardiothorac Vasc Anesth 2007;21(5):632-635

5 Reich DL, Mittnacht A, London M, Kaplan J. Monitoring of the heart and vascular system. In: Kaplan JA, Reich DL, Lake CL, Konstadt SN, eds. Kaplan's Cardiac Anaesthesia. Philadelphia, PA: Elsevier Saunders; 2006:386

6 Gödje O, Höke K, Goetz AE, et al. Reliability of a new algorithm for continuous cardiac output determination by pulsecontour analysis during hemodynamic instability. Crit Care Med 2002;30(1):52-58

7 Pittman J, Bar-Yosef S, SumPing J, Sherwood M, Mark J. Continuous cardiac output monitoring with pulse contour analysis: a comparison with lithium indicator dilution cardiac output measurement. Crit Care Med 2005;33(9):2015-2021

8 Chaney JC, Derdak S. Minimally invasive hemodynamic monitoring for the intensivist: current and emerging technology. Crit Care Med 2002;30(10):2338-2345

9 Rödig G, Prasser C, Keyl C, Liebold A, Hobbhahn J. Continuous cardiac output measurement: pulse contour analysis vs thermodilution technique in cardiac surgical patients. $\mathrm{Br} \mathrm{J}$ Anaesth 1999;82(4):525-530

10 Yamashita K, Nishiyama T, Yokoyama T, Abe H, Manabe M. Cardiac output by pulse CO is not interchangeable with thermodilution in patients undergoing OPCAB. Can J Anaesth 2005;52(5):530-534

11 Pearse RM, Ikram K, Barry J. Equipment review: an appraisal of the LiDCO plus method of measuring cardiac output. Crit Care 2004;8(3):190-195

12 de Vaal JB, de Wilde RB, van den Berg PC, Schreuder JJ, Jansen JR. Less invasive determination of cardiac output from the arterial pressure by aortic diameter-calibrated pulse contour. Br J Anaesth 2005;95(3):326-331

13 Mayer J, Boldt J, Schöllhorn T, Röhm KD, Mengistu AM, Suttner S. Semi-invasive monitoring of cardiac output by a new device 
using arterial pressure waveform analysis: a comparison with intermittent pulmonary artery thermodilution in patients undergoing cardiac surgery. Br J Anaesth 2007;98(2):176-182

14 Mayer J, Boldt J, Poland R, Peterson A, Manecke GR Jr. Continuous arterial pressure waveform-based cardiac output using the FloTrac/Vigileo: a review and meta-analysis. J Cardiothorac Vasc Anesth 2009;23(3):401-406

15 Chatti R, de Rudniki S, Marqué S, et al. Comparison of two versions of the Vigileo-FloTrac system (1.03 and 1.07) for stroke volume estimation: a multicentre, blinded comparison with oesophageal Doppler measurements. Br J Anaesth 2009; 102(4):463-469
16 Senn A, Button D, Zollinger A, Hofer CK. Assessment of cardiac output changes using a modified FloTrac/Vigileo algorithm in cardiac surgery patients. Crit Care 2009;13(2):R32

17 Maddali MM, Waje ND, Sathiya PM. Authentication of radial versus femoral arterial pressure waveform-derived cardiac output with transesophageal echocardiography-derived cardiac output measurements in patients undergoing on-pump coronary bypass surgery. J Cardiothorac Vasc Anesth 2017; 31(4):1183-1189 